\title{
THE A2667 GIANT ARC AT $z=1.03:$ EVIDENCE FOR LARGE-SCALE SHOCKS AT HIGH REDSHIFT
}

\author{
T.-T. YUAN ${ }^{1}$, L. J. KewLeY ${ }^{1,2}$, A. M. SWINBANK ${ }^{3}$, AND J. RICHARD ${ }^{4}$ \\ ${ }^{1}$ Institute for Astronomy, University of Hawaii, 2680 Woodlawn Drive, Honolulu, HI 96822, USA \\ ${ }^{2}$ Research School of Astronomy and Astrophysics, The Australian National University, Cotter Road, Weston Creek, ACT 2611, Australia \\ ${ }^{3}$ Institute for Computational Cosmology, Department of Physics, Durham University, South Road, Durham, DH1 3LE, UK \\ ${ }^{4}$ CRAL, Observatoire de Lyon, Université Lyon 1, 9 Avenue Ch. André, F-69561 Saint Genis Laval Cedex, France \\ Received 2012 June 13; accepted 2012 September 17; published 2012 October 17
}

\begin{abstract}
We present the spatially resolved emission line ratio properties of a $\sim 10^{10} M_{\odot}$ star-forming galaxy at redshift $z=1.03$. This galaxy is gravitationally lensed as a triple-image giant arc behind the massive lensing cluster A2667. The main image of the galaxy has magnification factors of $14 \pm 2.1$ in flux and $\sim 2 \times 7$ in area, yielding an intrinsic spatial resolution of 115-405 pc after adaptive optics correction with OSIRIS at KECK II. The Hubble Space Telescope morphology shows a clumpy structure and the $\mathrm{H} \alpha$ kinematics indicates a large velocity dispersion with $V_{\max } \sin (i) / \sigma \sim 0.73$, consistent with high-redshift disk galaxies of similar masses. From the $\left[\mathrm{N}_{\mathrm{II}}\right] / \mathrm{H} \alpha$ line ratios, we find that the central $350 \mathrm{pc}$ of the galaxy is dominated by star formation. The $[\mathrm{N}$ II] $/ \mathrm{H} \alpha$ line ratios are higher in the outer disk than in the central regions. Most noticeably, we find a blueshifted region of strong $[\mathrm{N} \mathrm{II}] / \mathrm{H} \alpha$ emission in the outer disk. Applying our recent $\mathrm{H}$ II region and slow-shock models, we propose that this elevated $[\mathrm{N} \mathrm{II]} / \mathrm{H} \alpha$ ratio region is contaminated by a significant fraction of shock excitation due to galactic outflows. Our analysis suggests that shocked regions may mimic flat or inverted metallicity gradients at high redshift.
\end{abstract}

Key words: galaxies: abundances - galaxies: evolution - galaxies: high-redshift - gravitational lensing: strong ISM: jets and outflows

Online-only material: color figures

\section{INTRODUCTION}

One of the most challenging aspects of current galaxy formation and evolution theory is the modeling of feedback processes associated with star formation and/or nuclear activities. Feedback is considered to be the key solution to many observational puzzles such as the quenching of star formation in massive galaxies (e.g., Bower et al. 2006; Dekel et al. 2009), the origin of the mass-metallicity relation (e.g., Tremonti et al. 2004; Finlator \& Davé 2008), the enrichment of the interstellar and intergalactic medium (ISM, IGM; e.g., Veilleux et al. 2005, and references therein), the flatter central dark matter profile than that predicted in $\Lambda$ CDM simulations (e.g., Flores \& Primack 1994; Governato et al. 2012), and the problem of the formation of bulgeless galaxies (e.g., Governato et al. 2010; Pilkington et al. 2011).

Galactic-scale winds and outflows are observed in a wide range of galaxy types that are undergoing significant star formation. Galactic-scale outflows have been frequently observed in (1) local starburst dwarf galaxies (Martin 1999; Kirby et al. 2011; van der Wel et al. 2011), (2) local ultra-luminous infrared galaxies (ULIRGs; Rupke et al. 2002, 2005; Martin 2005), and (3) high-redshift submillimeter galaxies (SMGs; Nesvadba et al. 2007; Alexander et al. 2010). Galactic winds are prevalent at intermediate and high redshifts (Shapley et al. 2003; Nesvadba et al. 2008; Sato et al. 2009; Weiner et al. 2009; Martin \& Bouché 2009; Martin et al. 2012; Rubin et al. 2010b, 2011).

In cosmological hydrodynamical simulations, implementation of a constant wind speed and outflow mass fails to reproduce the galaxy stellar mass functions (e.g., Crain et al. 2009). A momentum-driven wind feedback model is currently favored by observations and can reproduce the stellar mass function and the observed large-scale metal enrichment (Murray et al. 2005;
Oppenheimer \& Davé 2008; Finlator \& Davé 2008; Davé et al. 2011). In the momentum-driven wind model, the efficiency of the winds or mass-loading factor $\left(\dot{M}_{\text {wind }} / \mathrm{SFR}\right)$ scales with the declining power of the mass or circular velocity $\left(V_{c}^{-1}\right)$ of the galaxy $\left(\dot{M}_{\text {wind }} / \mathrm{SFR} \propto V_{c}^{-1}\right)$. However, whether this simple scaling relation applies to individual galaxies of a wide mass range at different redshifts remains to be tested. The physical picture of how exactly the winds interact with and recycle the energy and chemical elements to the ISM and IGM is still lacking. Indeed, Hopkins et al. (2012) have recently developed a more realistic stellar feedback model that includes a complex multi-phase structure that depends on the interaction between multiple feedback mechanisms operating on different spatial and temporal scales.

Note that past studies of gas inflows/outflows have mostly been based on slit spectra of a small number of individual galaxies (Sato et al. 2009; Rubin et al. 2010a) or average composite spectra from stacked low signal-to-noise $(\mathrm{S} / \mathrm{N})$ spectra (Weiner et al. 2009; Rubin et al. 2010b). Recent studies using KECK/LRIS data have significantly expanded the sample of individual galaxies and prevalent outflows have been confirmed for hundreds of individual galaxies at $z \sim 1$ (Rubin et al. 2012; Martin et al. 2012).

To provide a more concrete observational baseline for the sophisticated feedback models such as Hopkins et al. (2012), the next leap forward is to look for wind signatures in a spatially resolved manner. Integral field spectroscopic (IFS) observations are needed to separate the various spatial components of the multi-phased ISM (Arribas et al. 2001; Rich et al. 2010, 2011). Recent surveys of wide field-of-view (FOV) IFS on local galaxies have dramatically expanded the quantitative analysis for galactic-wide winds and shocks (Rich et al. 2010, 2011; Arribas et al. 2008; Sharp \& Bland-Hawthorn 2010). 
IFS studies have found widespread shock excitation associated with galactic outflows in massive local galaxies (Farage et al. 2010; Sharp \& Bland-Hawthorn 2010; Rich et al. 2010, 2011). Shocks are common products of winds and can also be induced by gas accretion and tidal flows in mergers (Veilleux et al. 2005; Farage et al. 2010; Genzel et al. 2011; Soto et al. 2012). Shock excitation contributes to the ionized state of the hot gas in galaxies, which also depends intimately on the chemical abundance of the ISM. To identify the location and fraction of shock excitation across an entire galaxy, it is crucial to disentangle the shock induced ionization and energy dissipation from other mechanisms.

Narrow line ratio maps can be used to probe the ionization nature of the sources (Veilleux et al. 2002; Veilleux \& Rupke 2002). Strong emission line ratios such as the BPT diagram (Baldwin et al. 1981; Veilleux \& Osterbrock 1987; Allen et al. 1998; Kewley et al. 2001b, 2006) remains one of the most practical methods to probe the ionization mechanisms at both low and high redshifts. Combined with the most recent photoionization and shock models, strong line ratios such as $[\mathrm{N}$ II $] / \mathrm{H} \alpha,[\mathrm{O} \mathrm{III}] / \mathrm{H} \beta$, and $[\mathrm{S} \mathrm{II}] / \mathrm{H} \alpha$ offer a powerful tool to identify the excitation mechanisms in non-obscured regions. For example, Rich et al. (2010) showed that in NGC 839, the $[\mathrm{N} I \mathrm{II}] / \mathrm{H} \alpha,[\mathrm{O} \mathrm{III}] / \mathrm{H} \beta$, and $\left[\mathrm{S}_{\mathrm{II}}\right] / \mathrm{H} \alpha$ ratios are systematically lower near the nucleus, yet high along an outflow bicone. The region with high line ratios is remarkably well fitted by a shock model with slow velocities typical of galactic winds.

At high redshift, the star formation rate (SFR) of galaxies is substantially larger than local star-forming (SF) galaxies (Noeske et al. 2007a, 2007b; Wuyts et al. 2011; Sobral et al. 2012b), and galactic outflows are prevalent and powerful (Erb et al. 2006; Swinbank et al. 2007; Weiner et al. 2009; Rubin et al. 2010b; Alexander et al. 2010; Genzel et al. 2011). It is therefore of paramount importance to observe the masses and energies of high-redshift winds to constrain the relations between the wind efficiency, SFR, and galaxy masses on a broader scale.

Because of the observational challenges, outflows and super winds at high redshift have mostly been reported for Lyman break galaxies, SMGs, or in quasar absorption line systems. Spatially resolved spectroscopy for high- $z$ galaxies has been limited to the very massive starburst galaxies (Cresci et al. 2010; Genzel et al. 2011). With seeing-limited observations, Cresci et al. (2010) reported three $z \sim 3 \mathrm{UV}$-selected galaxies with inverted metallicity gradients (i.e., lower metallicity in the nucleus than in the outer disk). The inverted gradient was explained by primordial cold gas being funneled to the nucleus. These studies demonstrate the power of integral field unit (IFU) studies in revealing the underlying physical processes of the gas accretion at high redshifts.

With the aid of magnification from gravitational lensing and adaptive optics (AO), we can begin to probe the details of fainter and less massive galaxies at high redshift (Nesvadba et al. 2008; Swinbank 2007; Jones et al. 2010b; Sharon et al. 2012; Wuyts et al. 2012). The highly magnified multiple images in massive clusters can be magnified by more than 10 , offering a rare opportunity to obtain spatially resolved observations. This technique has enabled the first high-resolution measurements of metallicity gradients at high redshift (Stark et al. 2008; Jones et al. 2010a; Yuan et al. 2011).

To determine the metallicity gradient evolutions with redshift, we are undertaking a metallicity gradient survey on lensed galaxies behind galaxy clusters. Here we report observations of the $z=1.03$ arc behind the massive cluster A2667. We find

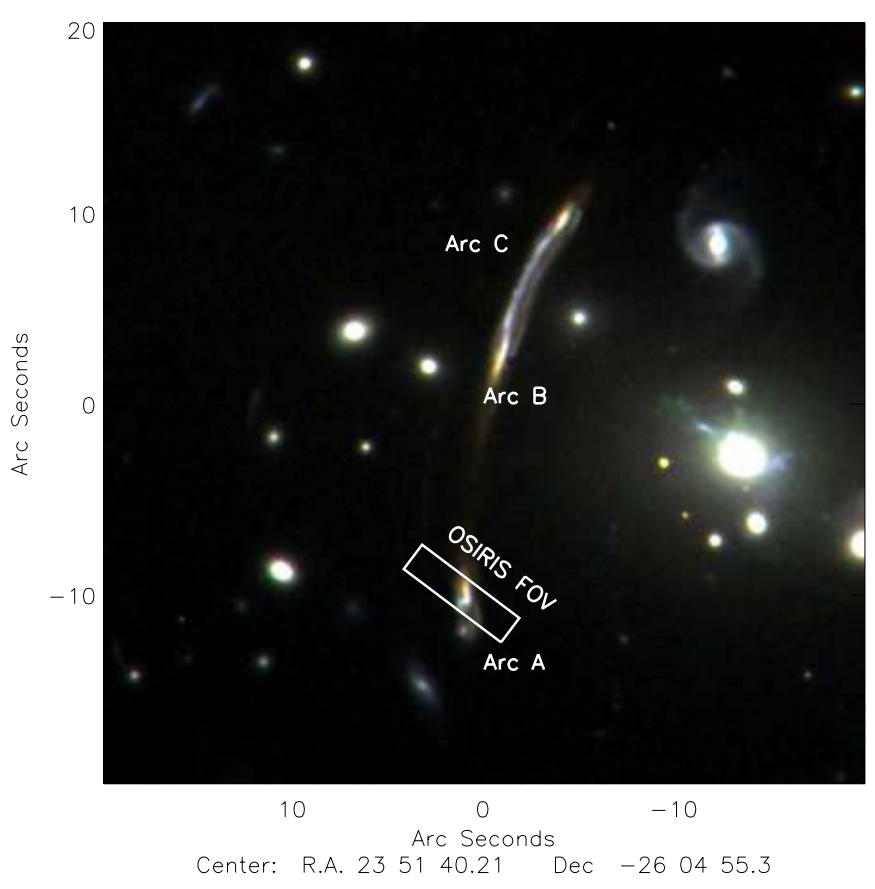

Figure 1. Three-color image of the A2667 giant arc at $z=1.03$ with HST F814W (red), F606W (green), and F450W (blue) filters. The white box on the bottom left shows the FOV and P.A. $\left(37^{\circ}\right)$ of the OSIRIS Jbb filter. The triple image is composed of $\operatorname{arcs} \mathrm{A}, \mathrm{B}$, and C. The IFU was centered on arc A $\left(\alpha_{2000}, \delta_{2000}\right)=(23: 51: 40.023,-26: 04: 51.66)$. North is up, and east is to the left.

(A color version of this figure is available in the online journal.)

anomalous spatial line ratio variations indicative of large-scale shocks.

Throughout this paper we use a $\Lambda$ CDM cosmology with $H_{0}=70 \mathrm{~km} \mathrm{~s}^{-1} \mathrm{Mpc}^{-1}, \Omega_{M}=0.30$, and $\Omega_{\Lambda}=0.70$. At $z=1.0,1$ arcsec corresponds to $8.1 \mathrm{kpc}$ and a look-back time of 9.3 gigayear (Gyr). We use solar oxygen abundance $12+$ $\log (\mathrm{O} / \mathrm{H})_{\odot}=8.66$ (Asplund et al. 2005).

\section{OBSERVATIONS, DATA REDUCTION, AND ANALYSIS}

\subsection{Observations}

The A2667 giant arc is a three-image lensing system. Covone et al. (2006) provided the spectroscopic redshift of $z=1.033$ for the arc and the lensing models of the A2667 cluster at $z=0.233$. We observed the A2667 giant arc with the OH-Suppressing Infra-Red Imaging Spectrograph (OSIRIS; Larkin et al. 2006) in natural guide star (NGS) AO mode on the KECK II telescope at $\left(\alpha_{2000}, \delta_{2000}\right)=(23: 51: 40.023,-26: 04: 51.66)$. The observation was conducted on the night of 2011 September 20-21, using NGS AO correction. The $R=14 \mathrm{mag}$ NGS was on the edge of the NGS FOV of OSIRIS, restricting the positional angle (P.A.) to $37^{\circ}$. The 100 mas pixel scale was used. Since only broadband filters were available on OSIRIS at the time, we used the Jbb filter (spectral coverage: 1.18-1.416 $\mu \mathrm{m}$, spectral resolution: $R \sim 3600$, FOV 1".6 × 6."4), which covers the $\mathrm{H} \alpha$, [N II], and $[\mathrm{S} \mathrm{II}]$ lines. The observations were conducted in a standard ABBA dithering sequence, with the A frame centered on the target and the $\mathrm{B}$ frame centered on an object-free sky region $\sim 20$ arcsec northeast of the target. The same position angle was used for A and B (sky) frames. A total of five exposures were obtained for the target, with $900 \mathrm{~s}$ on each individual frame. The net on-target exposure time is 75 minutes. Figure 1 shows the OSIRIS FOV and locations of the A2667 arc on the Hubble 


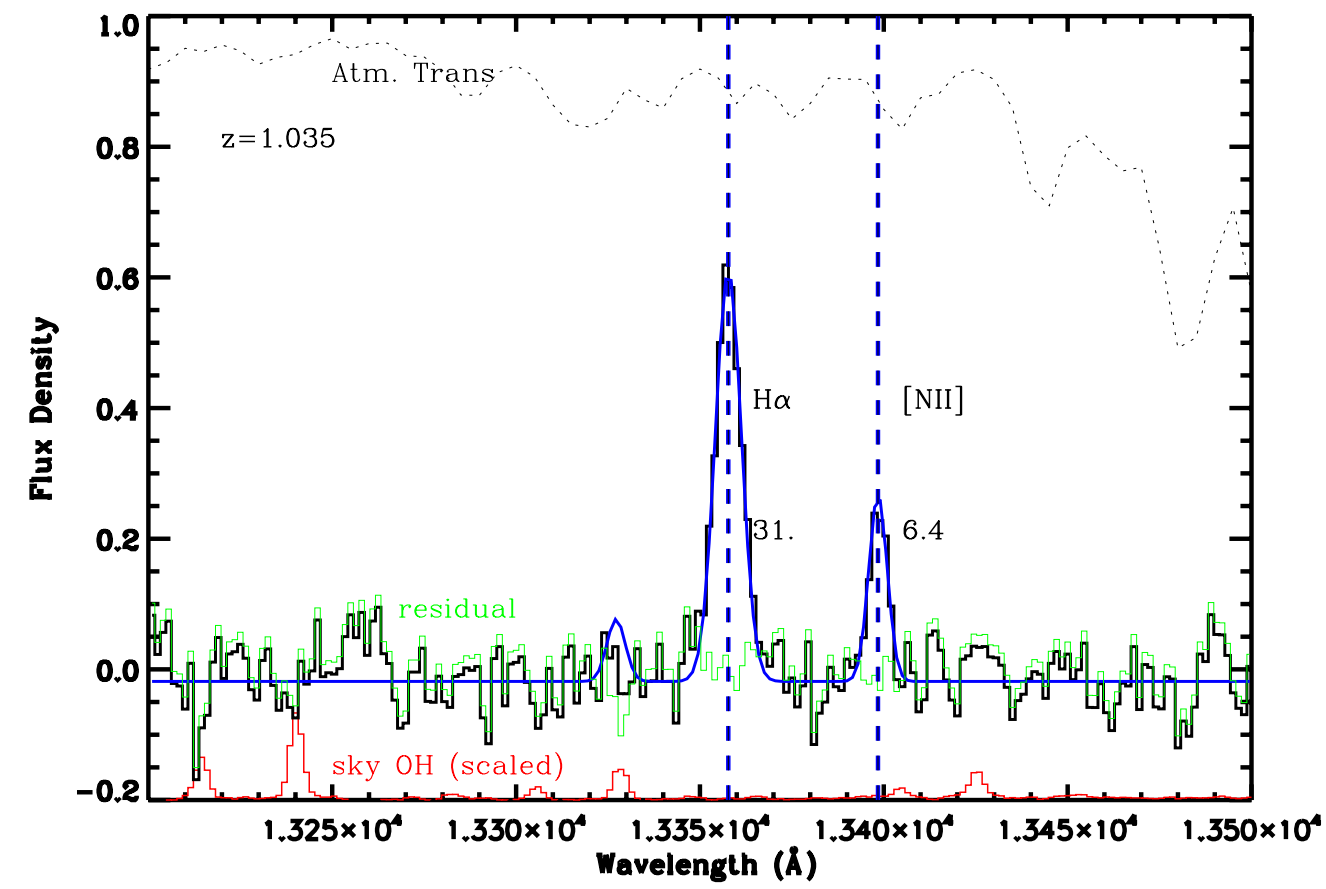

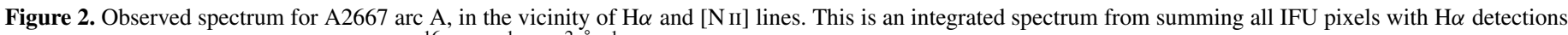

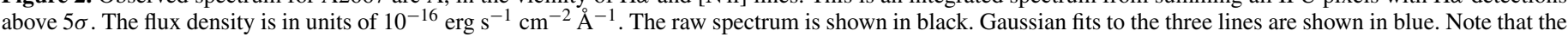

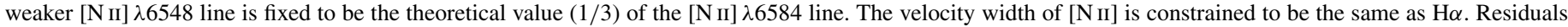

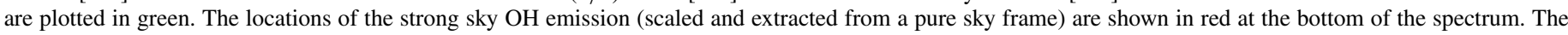

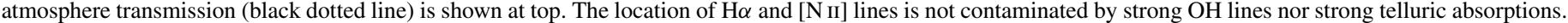
We achieve an $\mathrm{S} / \mathrm{N}$ of 37 on $\mathrm{H} \alpha$ and of 6.4 on [N II].

(A color version of this figure is available in the online journal.)

Space Telescope (HST) F814W, F606W, F450W color-coded image.

Before the science target exposure, short exposures of the tiptilt (TT) star were taken to center the IFU. An offset was applied from the TT star to the science target. The offset was determined from astrometry corrected HST images with $\sim 0$ '.25 (i.e., $\sim 2-3$ OSIRIS pixels) accuracy. Gaussian fitting to the point-spread function (PSF) of the TT star yields an average FWHM of $00^{\prime \prime} 13$. The average FWHM of the sky $\mathrm{OH}$ lines is measured to be $\sim 2.5 \AA$ for our data and we use this value as the instrumental resolution. In the analysis below, we deconvolve the line widths with this instrument resolution by subtracting the instrumental resolution in quadrature from the best-fitting Gaussian $\sigma$.

\subsection{Data Reduction}

Individual exposures were first reduced using the OSIRIS data reduction pipeline (Larkin et al. 2006). We used the sky subtraction IDL code of Davies (2007), which we have modified to optimize the sky subtraction specifically around the wavelength region of $\mathrm{H} \alpha$ and [N II]. The [S II] lines are undetected due to strong telluric absorption. Since we are mainly concerned with the emission lines, a first-order polynomial function was fit to the continuum for each spatial sample pixel (spaxel) and then the continuum was subtracted from each spaxel. The subtraction of the continuum helps to improve the removal of lenslet to lenslet variations. The final datacube was constructed by aligning the sub-exposures with the centroid of the $\mathrm{H} \alpha$ images and combining using a $3 \sigma$ mean clip to reject cosmic rays and bad lenslets. Telluric correction and flux calibration were performed by averaging three A0 standard stars (HIP220, HIP1272, HIP14719) observed immediately after the science exposure. Due to the low declination of this target, the airmass varied between 1.4 and 1.8 during the observation. The uncertainty in absolute flux calibration is estimated to be $\sim 20 \%-30 \%$. However, the relative flux ratio of $[\mathrm{N} \mathrm{II}] / \mathrm{H} \alpha$ is accurate to $10 \%$.

\subsection{Analysis}

Our analysis follows the procedures outlined in Jones et al. (2010b). Gaussian profiles were fitted simultaneously to the three emission lines: [N $\mathrm{NI}_{\mathrm{II}} \lambda 6548,6583$, and $\mathrm{H} \alpha$. The centroid and velocity width of $\left[\mathrm{N}_{\mathrm{II}}\right] \lambda 6548,6583$ lines were constrained by the velocity width of $\mathrm{H} \alpha \lambda 6563$, and the ratio of $[\mathrm{N}$ II $] \lambda 6548$ and $\left[\mathrm{N}_{\mathrm{II}}\right] \lambda 6583$ is constrained to be the theoretical value of Osterbrock (1989). We fit the line profiles using a weighted $\chi^{2}$ minimization procedure which takes into account the greater noise level close to atmospheric $\mathrm{OH}$ emission. A blank region of sky within each reduced datacube was used to determine the variance spectrum. Since the variance spectrum is dominated by sky emission, the fitted spectra were weighted by the inverse of the variance spectrum (dominated by sky counts) so that regions of strong skylines do not cause spurious fits. Fortunately, the sky emission is weak over the $\mathrm{H} \alpha$, [N II] wavelength range for A2667 arc A (Figure 2). We compute the $\chi^{2}$ statistic for the best-fitting Gaussian lines and compare with that of a featureless spectrum. We first fit the spectrum for each pixel, requiring a minimum of $5 \sigma$ for detection. Where this criterion is not met, we average the surrounding $3 \times 3$ spatial pixels to achieve higher $\mathrm{S} / \mathrm{N}$. No fit is made if the $3 \times 3$ averaging fails to produce the minimum $\mathrm{S} / \mathrm{N}$. Figure 2 shows the integrated spectrum from the image plane.

In order to check that our results are insensitive to the lensing model uncertainty, we first analyze the data on the image plane, and then repeat the analysis on the source plane. We find that 


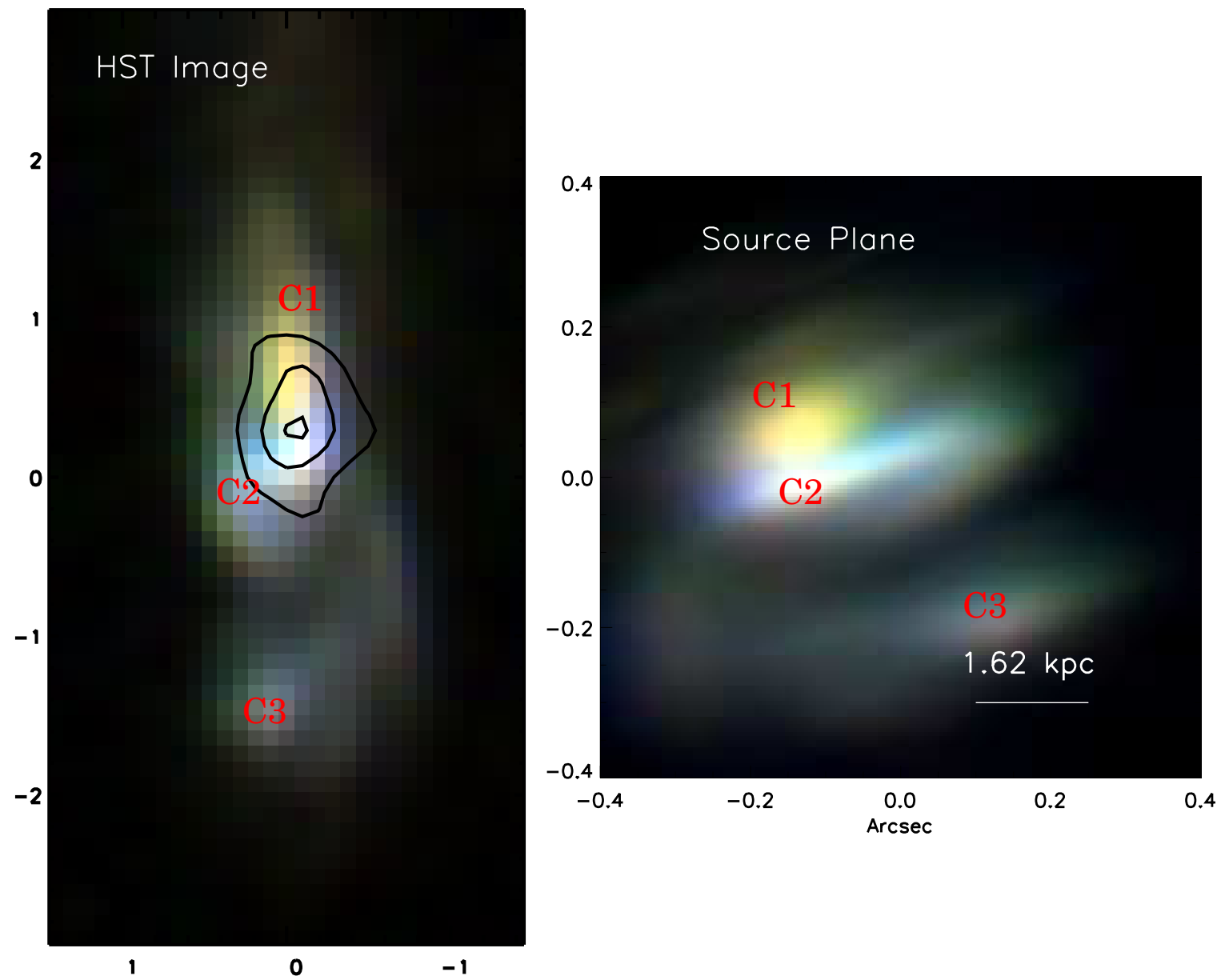

Figure 3. Left: HST F814W, F606W, F450W three-color image for A2667 arc A. The black contours show the observed H $\alpha$ flux levels of 2, 3, 3.5 in units of $10^{-17} \mathrm{erg} \mathrm{s}^{-1} \mathrm{~cm}^{-2} \operatorname{arcsec}^{-2}$. Right: reconstructed source-plane morphology from the three-color HST image on the left. The physical scale is marked. The distance between the center of the red and blue clumps is $\sim 600 \mathrm{pc}$. There are three main clumps revealed in both the observed HST and reconstructed images: a northern red component (marked as C1), and two blue southern clumps (marked as C2, C3). The morphology implies either a face-on clumpy disk or a merging system. North is up and east is to the right.

(A color version of this figure is available in the online journal.)

the results are consistent for the image-plane and source-plane analysis. In the following sections, we will show the spectra measured on the image plane with associated physical scales determined from the source plane.

\section{GLOBAL PROPERTIES OF THE GIANT ARC}

We determined the total photometry for arc A in seven bands from HST and Spitzer archival data: HST ACS/F814, WFPC2/F606W, WFPC2/F450W, and Spitzer IRAC 3.6, 4.5, 5.8 , and $8.0 \mu \mathrm{m}$. Chandra data are also available for the cluster, but we find no X-ray detection on the giant arc. We used a fixed aperture of diameter $4^{\prime \prime}$ defined in the ACS/F814 band to measure the photometry. For the IRAC colors, we measured the PSF near arc A using the bright isolated stars in the FOV and convolve the ACS/F814 aperture with this PSF to calculate the aperture flux. Note that the photometric aperture covers the entire image of arc A whereas the OSIRIS FOV does not cover the tip of the southern clump C3 (Figure 1 for OSIRIS FOV; C3 is labeled in Figure 3). This southern clump only contributes to $\sim 5 \%$ to the total photometry, well below the $\sim 10 \%$ photometric measurement error. The intrinsic photometric error is dominated by the lensing magnification uncertainty $(\sim 15 \%)$.

We use the software LE PHARE (Ilbert et al. 2010) to determine the stellar mass. LE PHARE is a photometric redshift and simulation package based on population synthesis models of Bruzual \& Charlot (2003). We choose the initial mass function (IMF) by Chabrier (2003) and the Calzetti et al. (2000) attenuation law, with $E(B-V)$ ranging from 0 to 2 and an exponentially decreasing SFR (SFR $\propto \mathrm{e}^{-t / \tau}$ ) with $\tau$ varying between 0 and 13 Gyr. After correcting for the flux magnification of $\mu=14 \pm 2.1$, the stellar mass from spectral energy distribution (SED) fitting is $M_{\text {star }}=1.90_{-1.0}^{+2.0} \times 10^{10} M_{\odot}$ (lensing uncertainty included), assuming the best-fit extinction value $E(B-V)=0.6$, and $\mathrm{SFR}_{\mathrm{SED}}=50 \pm 35 M_{\odot} \mathrm{yr}^{-1}$. Taking $E(B-V)_{\text {gas }}=E(B-V)_{\text {star }} / 0.44$ (Calzetti 1997) and $\mathrm{Rv}=3.1$, we obtain $A(\mathrm{H} \alpha)=3.46$ according to the Cardelli et al. (1989) classical extinction curve. Note that the $A(\mathrm{H} \alpha)$ is $\sim 2.46$ higher than the median $A(\mathrm{H} \alpha)(\sim 1.0)$ at similar masses from the local and $z \sim 1.7 \mathrm{SF}$ galaxy dust extinction versus stellar mass relation (Garn \& Best 2010; Sobral et al. 2012a).

The total $\mathrm{H} \alpha$ flux from our OSIRIS spectra is $6.3 \pm$ $0.2 \times 10^{-16} \mathrm{erg} \mathrm{s}^{-1} \mathrm{~cm}^{-2}$ (before correcting for lensing magnification). Applying the Kennicutt (1998) SFR prescription and after correcting for lensing magnification and extinction $(A(\mathrm{H} \alpha)=3.46)$, the $\mathrm{H} \alpha$ SFR is estimated to be $\mathrm{SFR}_{\mathrm{H} \alpha}=49.2 \pm$ $16.5 M_{\odot} \mathrm{yr}^{-1}$. Note that the error is calculated by assuming a $15 \%$ uncertainty in lensing magnification and $30 \%$ error in $\mathrm{H} \alpha$ flux measurement. The error estimation excludes the uncertainty in extinction and aperture flux loss due to the coverage 


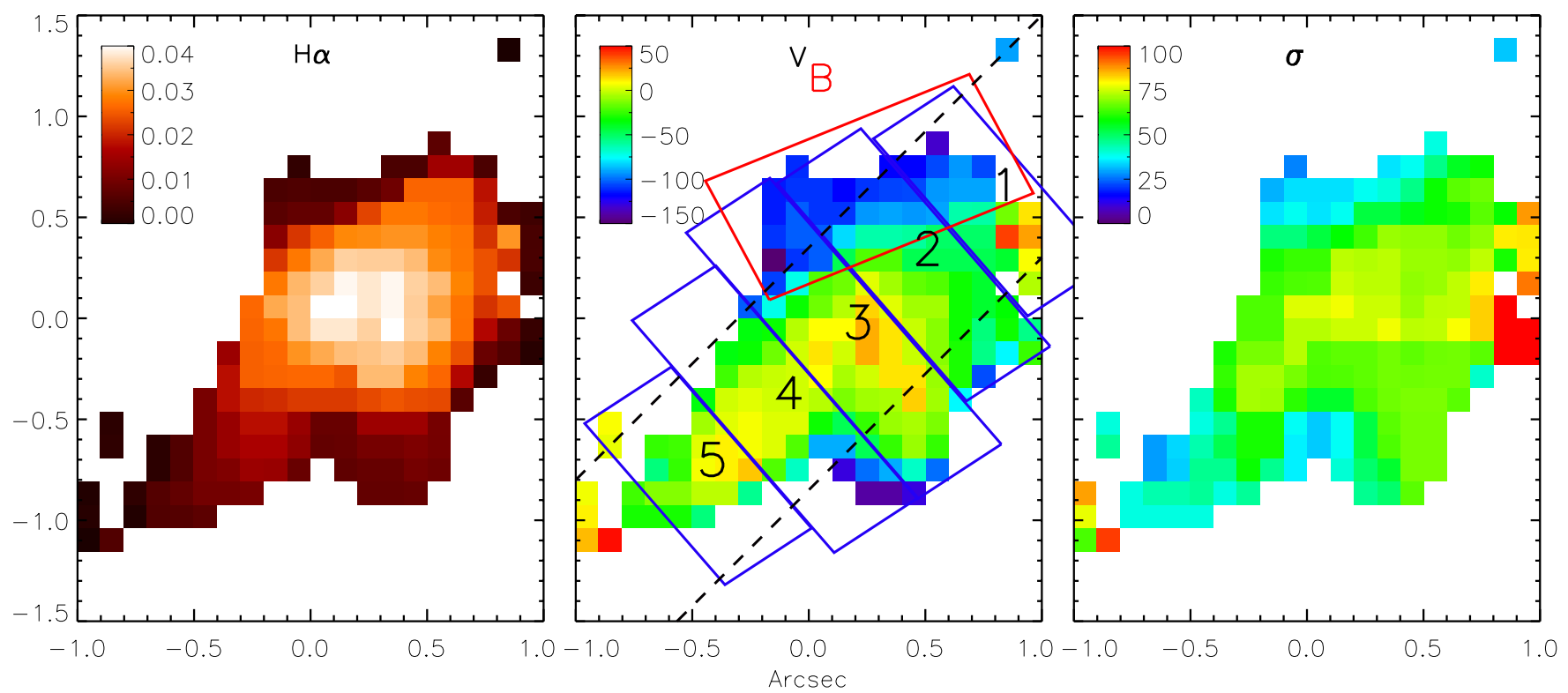

Figure 4. From left to right: the $\mathrm{H} \alpha$ intensity, $\mathrm{H} \alpha$ Gaussian fit velocities, and velocity dispersions presented on the image plane. The $\mathrm{H} \alpha$ flux is in units of $10^{-17} \mathrm{erg} \mathrm{s}^{-1} \mathrm{~cm}^{-2}$, and the velocity is in unit of $\mathrm{km} \mathrm{s}^{-1}$. For the middle panel: the black dashed lines indicate the "slit" used for extracting the 1D spectrum in Figure 5; the blue and red boxes are used in the analysis of Section 5.2. These images are shown in IFS datacube coordinates. North and east are $37^{\circ}$ clockwise from the HST images in Figures 1 and 3.

(A color version of this figure is available in the online journal.)

Table 1

Derived Global Physical Properties for Arc A

\begin{tabular}{lccccccc}
\hline \hline $\begin{array}{l}\mathrm{Re} \\
\mathrm{kpc})\end{array}$ & $\begin{array}{c}E(B-V) \\
\mathrm{SED} \mathrm{Fit}\end{array}$ & $\begin{array}{c}\mathrm{SFR}_{\mathrm{H} \alpha} \\
\left(M_{\odot} \mathrm{yr}^{-1}\right)\end{array}$ & $\begin{array}{c}M_{\text {star(SED) }} \\
\left(10^{10} M_{\odot}\right)\end{array}$ & $\begin{array}{c}\sigma \\
\left(\mathrm{km} \mathrm{s}^{-1}\right)\end{array}$ & $\begin{array}{c}V_{\max } \sin i \\
\left(\mathrm{~km} \mathrm{~s}^{-1}\right)\end{array}$ & $\begin{array}{c}12+\log (\mathrm{O} / \mathrm{H}) \\
\text { Integrated }\end{array}$ & $\begin{array}{c}\mu, \mu 1, \mu 2 \\
\text { Flux, axis1, axis2 }\end{array}$ \\
\hline $1.1 \pm 0.2$ & 0.6 & $49.2 \pm 16.5$ & $1.90_{-1.0}^{+2.0}$ & $65 \pm 6$ & $48 \pm 12$ & $8.606 \pm 0.051$ & $14 \pm 2.1,2.0 \pm 0.4,7.0 \pm 1.4$ \\
\hline
\end{tabular}

Notes. Derived intrinsic global properties for A2667 arc A. Column 1: the half-light radius from fitting a Sérsic profile to the red HST component. Column 2: extinction derived from SED fitting. Column 3: SFR from $\mathrm{H} \alpha$ flux, corrected for lensing magnification and extinction. The error comes from the uncertainty in magnification and flux calibration. Column 4: stellar mass from SED fitting. Column 5: median velocity dispersion, weighted by $\mathrm{H} \alpha$ flux. Column 6: $V_{\max } \sin i$, see the text for methodology. Column 7: metallicity from the integrated spectrum. Column 8: flux magnification and size magnification in the major and minor axes.

of the OSIRIS instrument. The physical properties of Arc A are summarized in Table 1.

\section{LENSING RECONSTRUCTION, MORPHOLOGY, AND KINEMATICS}

\subsection{Lens Modeling}

We use the best-fit lensing model parameters from Covone et al. (2006) and Richard et al. (2010) and use the software Lenstoo ${ }^{5}$ (Kneib et al. 1993; Jullo et al. 2007) to derive the geometrical transformation between image-plane coordinates and source-plane coordinates. The HST image and IFU datacube are reconstructed using this mapping and based on the conservation of surface brightness. The total magnification is computed by the ratio of the sizes (or equivalently, the total fluxes) between the image and its source-plane reconstruction. As the magnification is not isotropic, the angular size of each image is more highly stretched along a specific axis. However, within the single image of $\operatorname{arc} \mathrm{A}$, the distortions among each pixel are identical. Figure 3 shows the reconstructed image from the HST F814W, F606W, F450W three-color image. The total flux magnification for $\operatorname{arc} \mathrm{A}$ is $\mu=14.0 \pm 2.1$, and linear magnifications in two axes are $\mu 1=7.0 \pm 1.4$ and $\mu 2=2.0 \pm 0.4$.

\footnotetext{
5 http://www.oamp.fr/cosmology/lenstool
}

\subsection{Morphology and Kinematics}

The HST three-color image reveals at least three main clumps (labeled as $\mathrm{C} 1, \mathrm{C} 2, \mathrm{C} 3$ ) for arc A. These three clumps are easily identified in both the image-plane (Figure 3, left) and sourceplane (Figure 3, right) morphology. The northern red clump (C1) could be dominated by an old stellar population. Alternatively, $\mathrm{C} 1$ could be heavily reddened by dust. After masking out the two blue clumps $(\mathrm{C} 2, \mathrm{C} 3)$ on the south, a Sérsic profile fit to the red component on the source plane yields a Sérsic index of $0.63 \pm 0.30$, and a half-light radius of $\operatorname{Re}=1.1 \pm 0.2 \mathrm{kpc}$. The blue clumps (C2, C3) are likely to be active SF regions. The peak of the H $\alpha$ emission is close to the largest blue clump as shown in the $\mathrm{H} \alpha$ contours of Figure 3. On the one hand, the asymmetric clumpy structure of A2667arc is similar to the asymmetric clumpy feature seen in $z \sim 2$ disks (Genzel et al. 2006, 2011; Law et al. 2009; Lehnert et al. 2009; Förster Schreiber et al. 2009, 2011). On the other hand, the morphology could also be interpreted as a merging system. We combine the morphology with the velocity structure to further analyze the disk-versus-merger origin of the system.

In Figure 4, we show the two-dimensional $\mathrm{H} \alpha$ intensity, $\mathrm{H} \alpha$ Gaussian fit velocity, and velocity dispersion maps. In Figure 5, we extract the one-dimensional (1D) velocities and dispersions from a slit oriented along the direction of the blue and red 


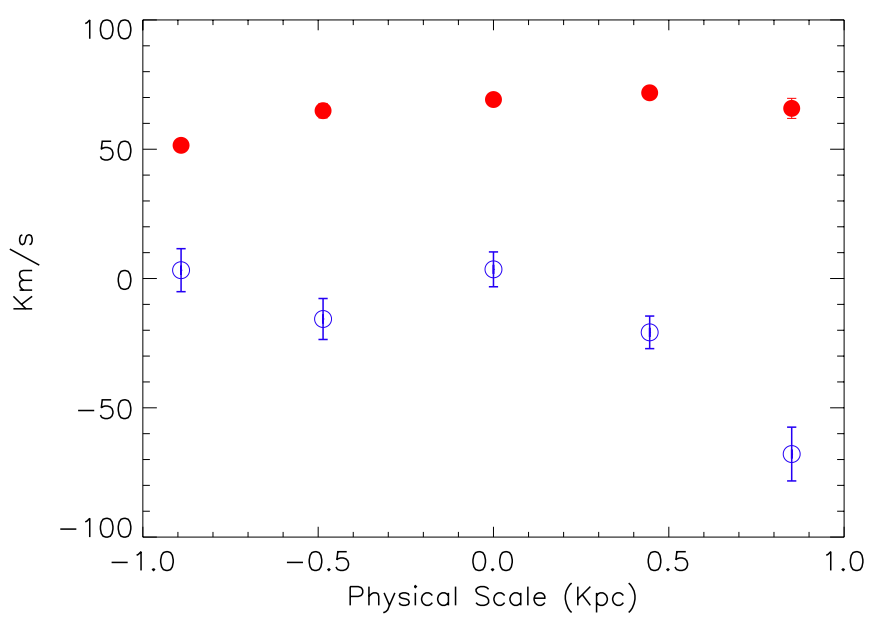

Figure 5. One-dimensional velocity extracted from the slit position shown in Figure 4. The five data bins from left to right correspond to the direction from Box 5 to Box 1 in Figure 4. The physical scale is calculated on the source plane. Note that the error bars on each data point are the bootstrapped median errors for all the pixels in the bins, weighted by the $\mathrm{H} \alpha$ intensity. The high velocity dispersion is consistent with those of turbulent high-redshift galaxy disks.

(A color version of this figure is available in the online journal.)

components (black dotted lines in Figure 4, middle panel). We divide the spaxels into five bins equally along the slit direction and extract the $\mathrm{H} \alpha$ flux weighted spectrum for each bin. There is a small velocity gradient $\left(\sim 75 \mathrm{~km} \mathrm{~s}^{-1}\right)$ across a $2 \mathrm{kpc}$ length. Since the shape of the $1 \mathrm{D}$ velocity curve is not clearly of a rotating disk, the small velocity gradient could result from either an almost face-on rotating field or a non-rotating system. The system however is clearly dominated by dispersion with $\sigma_{\text {median }} / V_{\max } \sin i \sim 1.3$, consistent with the large velocity dispersion of the high-redshift turbulent disks (Lehnert et al. 2009; Förster Schreiber et al. 2009; Jones et al. 2010b).

From our morphology and velocity maps, it remains unclear whether the A2667 arc is a face-on clumpy disk or a merging system, especially considering that the $\mathrm{H} \alpha$ lines are not observed across the entire galaxy. Higher $\mathrm{S} / \mathrm{N}$ data with better spatial coverage are required to more reliably model the velocity field.

\section{SPATIALLY RESOLVED LINE RATIOS}

\section{1. [N II] $\lambda 6583 / H \alpha$ as an Indicator for Metallicity and Non-photoionized Emission}

The $\left[\mathrm{N}_{\mathrm{II}}\right] \lambda 6583 / \mathrm{H} \alpha$ ratio is often used as an indicator for nebular metallicity at high redshift (up to $z \sim 2.5$ ). The adjacency of the two lines in wavelength means the line ratio is insensitive to reddening correction and flux calibration. The calibration of Pettini \& Pagel (2004, PP04) is commonly used to convert $[\mathrm{NII}] / \mathrm{H} \alpha$ to metallicity. Using the oxygen abundance of local H II regions, PP04 measured metallicities from the $T e$ (electron temperature) method and determined the correlation between metallicity and the $N 2(\equiv \log ([\mathrm{N} \mathrm{II}] \lambda 6583 / \mathrm{H} \alpha))$ values as follows:

$$
12+\log (\mathrm{O} / \mathrm{H})=8.90+0.57 \times N 2 .
$$

Note that at high abundance, PP04 used the strong line metallicities rather than the $T e$ metallicities because of the weakness of the $T e$-sensitive [O III] $\lambda 4363$ line. Strictly speaking, the PP04 relation only holds true for the range of $-1.8 \leqslant N 2 \leqslant-0.8$ (or $\left.0.0158 \leqslant\left[\mathrm{~N}_{\mathrm{II}}\right] / \mathrm{H} \alpha \leqslant 0.158\right)$. At extremely low $N 2$ values, the calibration of $N 2$ is difficult due to the weakness of the [N II] line. At $N 2>-0.8$, the relation is nonlinear. The reason for this is, first, as the metallicity becomes larger and $T e$ decreases, the production of secondary nitrogen begins to dominate and maintain a high value of $N 2$. At even higher metallicity, the rise in metallicity cools the nebula, lowering $T e$ and allowing fewer collisional excitations of the [N II] lines (Kewley \& Dopita 2002). We note that the $[\mathrm{N} \mathrm{II}] / \mathrm{H} \alpha$ ratio is particularly sensitive to non-thermal excitation. The presence of shock excitation or an active galactic nucleus (AGN) will increase the $[\mathrm{N} \mathrm{II}] / \mathrm{H} \alpha$ ratio and cause abundances determined to be artificially high (Baldwin et al. 1981; Kewley \& Dopita 2002; Rich et al. 2010). For metallicity studies, it is crucial to check if the $N 2$ values are within the regime of pure star formation.

On the other hand, if $N 2$ surpasses the linear metallicity diagnostic range, the high $N 2$ ratios may be a smoking gun for the presence of non-photoionization mechanisms such as shocks. For example, Veilleux \& Rupke (2002) studied the [N II]/H $\alpha$ distribution in the local super-wind galaxy M82. They found that for $[\mathrm{N} \mathrm{II}] / \mathrm{H} \alpha>0.5$, additional excitation mechanisms such as shocks are needed to explain the enhanced $[\mathrm{N}$ II $] / \mathrm{H} \alpha$ ratio. Using the most recent shock and photoionization models with IFU observations, Rich et al. (2011) showed that for the local galaxy IC 1623 , shocks contribute more than $40 \%$ of the line ratio of $[\mathrm{N}$ II $] / \mathrm{H} \alpha$.

\section{2. $[N \mathrm{II}] / \mathrm{H \alpha}$ in Boxed Regions}

To determine the spatial variation (if any) of metallicity or ionization sources, we analyze the spatial distribution of $[\mathrm{NII}] / \mathrm{H} \alpha$ ratios. In order to enhance the $\mathrm{S} / \mathrm{N}$ of the $N 2$ line ratio map, we bin the pixels in five rectangular regions along the major axis and extract spectra for each boxed region (Figure 4). The widths of the regions are chosen in such a way that each region contains sufficient pixels to extract a reliable spectrum (i.e., $\mathrm{S} / \mathrm{N}>3$ for the $[\mathrm{N}$ II] line). We derive the spectrum for each region using the $\mathrm{H} \alpha$ flux weighted median of all pixels in that region. We fit the $[\mathrm{N}$ II] and $\mathrm{H} \alpha$ emission lines and obtain the errors on $[\mathrm{N}$ II] $/ \mathrm{H} \alpha$ by propagating the flux errors of [N $\mathrm{II}]$ and $\mathrm{H} \alpha$.

The peak of the $\mathrm{H} \alpha$ emission corresponds to the center of Box 3 in Figure 4. We do not find significantly enhanced $[\mathrm{N}$ II $] / \mathrm{H} \alpha$ ratios in the central pixels and rule out AGN contamination of this target.

Our $[\mathrm{N} I \mathrm{II}] / \mathrm{H} \alpha$ emission line ratios are shown in Figure 6. The $[\mathrm{N}$ II $] / \mathrm{H} \alpha$ ratios of Boxes 3 and 4 are $0.303 \pm 0.034$ and $0.312 \pm 0.033$, respectively, close to solar values. The $[\mathrm{N} \mathrm{II}] / \mathrm{H} \alpha$ ratios for Boxes 1 and 2 are $0.472 \pm 0.046$ and 0.384 \pm 0.03 , respectively, significantly higher than those of Box 3 and Box 4. We can only derive an upper limit of $[\mathrm{N} \mathrm{II}] / \mathrm{H} \alpha(<0.233)$ for Box 5. The high [N II] $/ \mathrm{H} \alpha$ values in the non-central regions of the galaxy indicate either extremely high metallicity or the presence of non-photoionization excitation.

We further analyze individual pixels in Boxes 1 and 2 and find that the $[\mathrm{N}$ II $] / \mathrm{H} \alpha$ ratio is the highest in a sub-region highlighted as Box B in Figure 4. Very interestingly, Box B, which has the highest $[\mathrm{N}$ II $] / \mathrm{H} \alpha$ ratio $([\mathrm{N} \mathrm{II}] / \mathrm{H} \alpha=0.499 \pm 0.05)$, has also the "bluest" velocity ( $\Delta v \sim 150 \mathrm{~km} \mathrm{~s}^{-1}$ ) with respect to the center of Box 3. Unfortunately, the data quality is not sufficient to reveal any significant variations in the velocity dispersion map. As discussed in the previous section, large [N II] $/ \mathrm{H} \alpha$ ratios may indicate non-photoionized emission and therefore we do not convert this ratio to metallicity. We note that the consequences of deriving metallically using [ $\mathrm{N}$ II] $/ \mathrm{H} \alpha$ ratios in this case will result in the conclusion of a flat or inverted metallicity gradient 

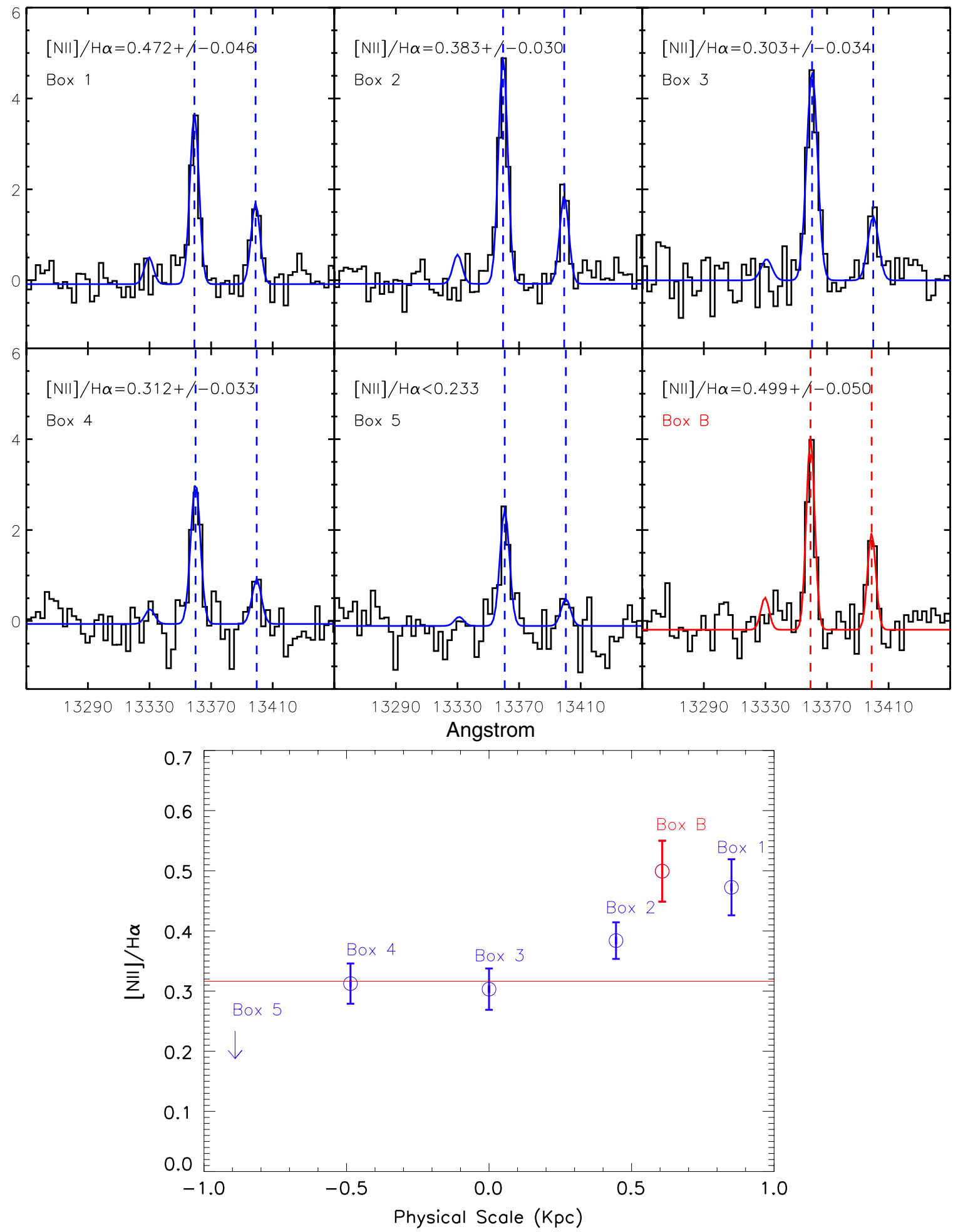

Figure 6. $[\mathrm{N}$ II] $/ \mathrm{H} \alpha$ ratio variation in boxed regions along the major axis. The top six panels show the observed image-plane spectra in vicinity of the $[\mathrm{N}$ II] and $\mathrm{H} \alpha$ for the six boxed regions in Figure 4. The spectra are the H $\alpha$ flux weighted median of the pixels in each box. The $x$ axes are wavelengths in $\AA$, and the $y$ axes are flux

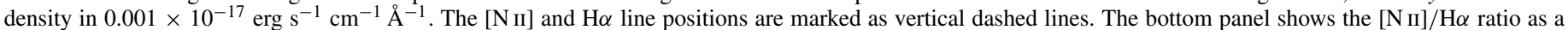
function of the intrinsic physical scale (after lensing correction) of the galaxy. The red horizontal line indicates the line ratio corresponding to the solar metallicity value. Ratios above solar cannot be directly related to metallicity. We also note a significant enhancement of [N II]/H $\alpha$ in Box B, a blueshifted region with respect to the galactic center.

(A color version of this figure is available in the online journal.) 


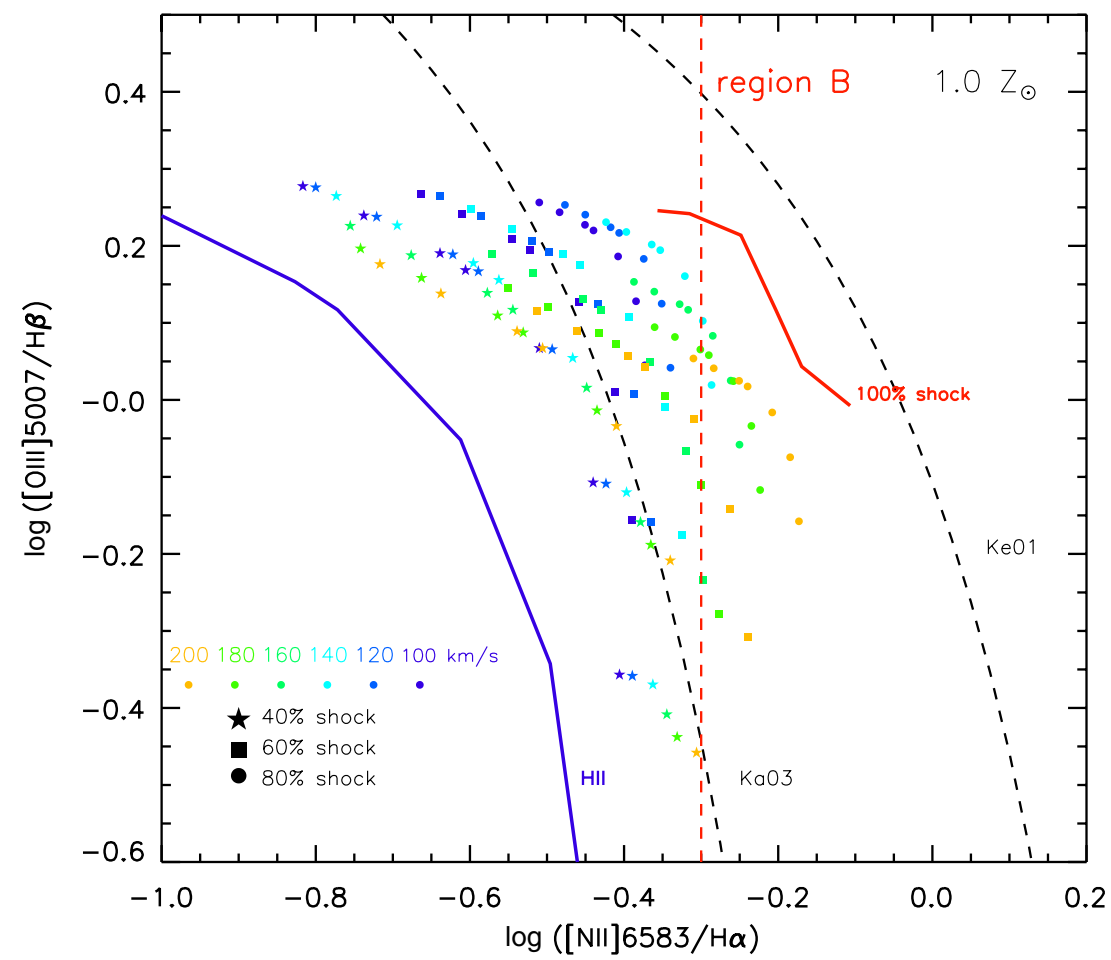

Figure 7. Starburst-shock mixing grids on the $[\mathrm{O} \mathrm{III}] / \mathrm{H} \beta$ vs. $[\mathrm{N} \mathrm{II}] / \mathrm{H} \alpha$ diagram. The dashed curve labeled as "Ke01" is the theoretical "maximum starburst line" derived by Kewley et al. (2001a) as an upper envelope for star-forming galaxies. The dashed curve labeled as "Ka03" is the Kauffmann et al. (2003) semi-empirical boundary for star-forming galaxies. The pure H II region models are shown as the blue solid line and the pure shock models are shown as the red solid line. The colored dots in between form a mixing sequence from pure $\mathrm{H}$ II region to pure shock excitation. Different colors represent different shock velocities and the relative contribution of shock is shown in different symbols. Solar abundance is adopted. The [N $\mathrm{II}] / \mathrm{H} \alpha$ ratio for region B in Figure 6 is highlighted as the vertical red dashed line.

(A color version of this figure is available in the online journal.)

(Appendix). In the next section, we discuss the possible origins of these elevated $[\mathrm{N} \mathrm{II}] / \mathrm{H} \alpha$ ratios.

\section{DISCUSSION}

The standard BPT diagrams prove to be a powerful tool for investigating the ionization sources in galaxies (Baldwin et al. 1981; Veilleux \& Osterbrock 1987; Kewley et al. 2001a, 2006; Kauffmann et al. 2003). Employing IFS data on the BPT diagram has revolutionized our understanding of the power sources across entire galaxies (e.g., Rich et al. 2010, 2011, 2012; Sharp \& Bland-Hawthorn 2010). The [N II]/H $\alpha$ versus $[\mathrm{O} \mathrm{III}] / \mathrm{H} \beta$ diagram is useful for distinguishing pure $\mathrm{SF}$ regions from non-photoionized regions. The $[\mathrm{N}$ II $] / \mathrm{H} \alpha$ ratio increases linearly with nebular metallicity until high metallicities where the $\log ([\mathrm{N} \mathrm{II}] / \mathrm{H} \alpha)$ ratio saturates at -0.5 (Denicoló et al. 2002; Kewley \& Dopita 2002; Pettini \& Pagel 2004). Any non-thermal contribution will shift the $[\mathrm{N} \mathrm{II}] / \mathrm{H} \alpha$ ratio toward higher values than this saturation level (Kewley et al. 2006; Rich et al. 2010, 2011). The $\log ([\mathrm{N} \mathrm{II}] / \mathrm{H} \alpha)$ ratio of region $\mathrm{B}$ is 0.2 dex larger than the saturation level of -0.5 , suggestive of shock ionization.

We apply a mixed grid of photoionization and shock models to quantify the potential relative contribution of shock and H II region ionization to the $[\mathrm{N}$ II $] / \mathrm{H} \alpha$ ratio. The $\mathrm{H}$ II region models are taken from the most recent Starburst99(SB99)/Mappings III model suite (Leitherer et al. 1999; Sutherland et al. 1993). The model setup and input parameters are similar to those of Levesque et al. (2010) except that we use a slightly higher mass cut of $120 M_{\odot}$ for the Salpeter IMF (Salpeter 1955). We use a continuous star formation history with age 5.0 Myr and $n_{e}=100 \mathrm{~cm}^{-3}$. Solar metallicity was used with a range of ionization parameters $\log \mathcal{U}(-3.5$ to -1.9$)$. We then mix the $\mathrm{H}$ II region model with the slow-velocity shock model as described in Sutherland et al. (1993), Farage et al. (2010), and Rich et al. $(2010,2011)$. We assume a $100 \%$ pre-ionization, with a slowshock velocity ranging from 100 to $200 \mathrm{~km} \mathrm{~s}^{-1}$, in increments $20 \mathrm{~km} \mathrm{~s}^{-1}$. These shock velocities are typical of galactic winds (Shopbell \& Bland-Hawthorn 1998; Rich et al. 2010, 2011; Sharp \& Bland-Hawthorn 2010).

Our shock and starburst models are shown in Figure 7 . We show mixing lines to represent the relative contributions of starbursts and shocks from $0 \%$ to $100 \%$. Shocks are able to produce higher $[\mathrm{N} \mathrm{II}] / \mathrm{H} \alpha$ ratios than pure starburst models for a given metallicity. According to our models, a $20 \%$ shock contribution could increase the $\log ([\mathrm{N} \mathrm{II}] / \mathrm{H} \alpha)$ value by 0.2 dex. The region $\mathrm{B}$ of the $\mathrm{A} 2667 \operatorname{arc}$ has $\log ([\mathrm{N} \mathrm{II}] / \mathrm{H} \alpha)=-0.3$, lying well within the region covered by the shock models.

Shocks induced by galactic winds/outflows associated with either stellar winds, supernovae or nuclear activity are commonly observed in local galaxies (e.g., Veilleux et al. 2005; Rupke et al. 2005; Rich et al. 2010; Soto \& Martin 2012). The ubiquity of winds is even more prominent at high redshift given their violent star formation activities (e.g., Pettini et al. 2001; Swinbank et al. 2005; Sato et al. 2009; Weiner et al. 2009; Martin \& Bouché 2009; Martin et al. 2012; Rubin et al. 2010b, 2011; Alexander et al. 2010; Steidel et al. 2010). In the cold flow scenario, the turbulent disks are associated with shocks (Genzel et al. 2011). The coincidence of region B being blueshifted with respect to the center strengthens the argument that it is an outflow shocked region.

Indeed, the outflow scenario is further supported by the detection of a broad component in $\mathrm{H} \alpha$. We detected a broad 


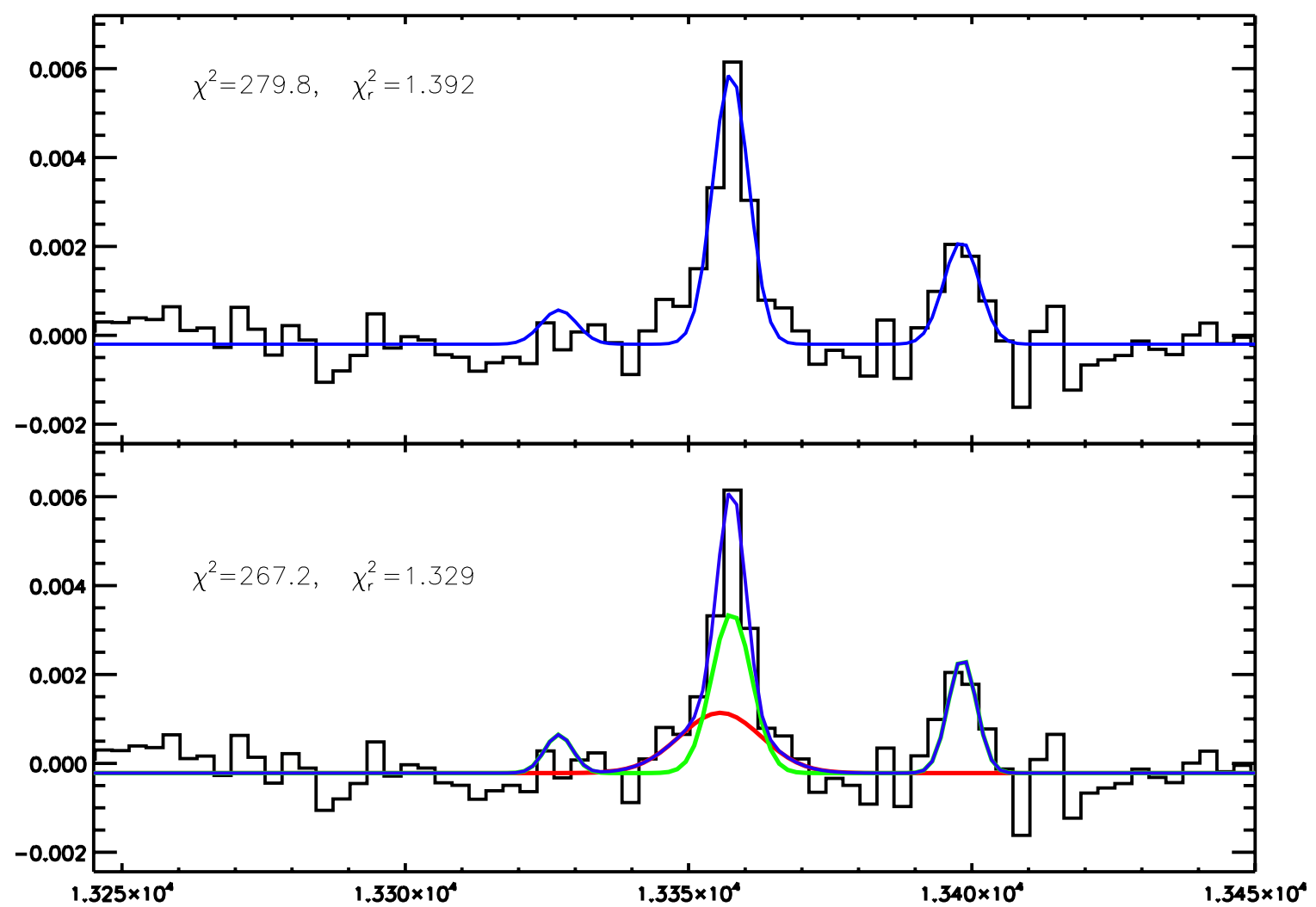

Figure 8. Co-added spectrum of Boxes 1, 2, 4, 5 (i.e., non-nuclear regions) in Figure 6. The top panel fits a single component to the H $\alpha$ and [N II] lines (blue solid curve). The bottom panel fits a narrow component (green curve) to the $\mathrm{H} \alpha,[\mathrm{NII}]$ lines and adds a broad component (red curve) to the $\mathrm{H} \alpha$ line; the sum of the two-component fit is shown (blue curves). The broad $\mathrm{H} \alpha$ component on the bottom panel (red line) is $\sim 60 \mathrm{~km} \mathrm{~s}^{-1}$ blueshifted from the narrow-line component.

(A color version of this figure is available in the online journal.)

component in the co-added spectrum of Boxes 1, 2, 4, 5. In Figure 8 we show that by including a broad component in $\mathrm{H} \alpha$, the fitting to the co-add spectrum is improved by $3 \sigma$ in a $\chi^{2}$ sense. The broad component indicates a broadening wing of $\Delta v(\mathrm{FWHM}) \sim 380 \pm 130 \mathrm{~km} \mathrm{~s}^{-1}$, with a slight blueshift of $\sim 60 \mathrm{~km} \mathrm{~s}^{-1}$ with respect to the narrow-line component. The contribution of the broad wing to the total $\mathrm{H} \alpha$ flux of the coadded spectrum is $f_{\text {broad }} \sim 40 \%$.

Because of the unknown orientation of the A2667arc system, the outflow velocity is difficult to estimate. We thus compare the outflow properties of the A2667arc with other studies using the observed line width $\Delta v(\mathrm{FWHM})$. For example, NGC 1569, a local dwarf starburst galaxy, has a wind-excited broad component of $\Delta v$ (FWHM) $\leqslant 300 \mathrm{~km} \mathrm{~s}^{-1}$ (Westmoquette et al. 2007). Arp 220, a famous local IR-luminous starburst galaxy, has a nuclear wind broadening of $\Delta v(\mathrm{FWHM}) \sim 300-800 \mathrm{~km} \mathrm{~s}^{-1}$ (Arribas et al. 2001). For a large sample of 39 local ULIRGs studied in Soto et al. (2012), the median line width for the shocked components is $\Delta v(\mathrm{FWHM}) \sim 339 \mathrm{~km} \mathrm{~s}^{-1}$ (after converting their $\sigma$ to FWHM using FWHM $=2.3548 \times$ Gaussian $\sigma$ ). The median SFR of the Soto et al. (2012) ULIRGs sample is $\sim 73 M_{\odot} \mathrm{yr}^{-1}$. At very high redshift $(z>2)$, the UV-selected massive SINS galaxies are found to have $\Delta v(\mathrm{FWHM}) \sim$ $500 \mathrm{~km} \mathrm{~s}^{-1}$ (Shapiro et al. 2009; Genzel et al. 2011), with a median SFR of $\sim 72 M_{\odot} \mathrm{yr}^{-1}$. Roughly, the broadening line width $\left(\Delta v(\mathrm{FWHM}) \sim 380 \pm 130 \mathrm{~km} \mathrm{~s}^{-1}\right)$ and SFR $(49.2 \pm$ $16.5 \mathrm{M}_{\odot} \mathrm{yr}^{-1}$ ) of A2667arc are most similar to the local ULIRGs sample of Soto et al. (2012). However, the similarity between the line widths and SFR of the A2667arc and the $z \sim 2$ SINS galaxies cannot be ruled out within $3 \sigma$ of the $\Delta v(\mathrm{FWHM})$ measurement uncertainty.
If, on the other hand, the high extranuclear [N II] $/ \mathrm{H} \alpha$ ratio regions in the A2667arc are caused by extremely high metallicity, we lack theories to explain how outer regions with supersolar metallicity would evolve spatially as a function of time. It is unclear how the A2667arc has gone through such a quick enrichment. Throughout the study we use the peak of $\mathrm{H} \alpha$ as the "galactic center," which does not necessarily concur with the true galactic center. However, the choice of center would not change our main conclusion that there is a shocked region blueshifted from the peak of $\mathrm{H} \alpha$ emission.

Finally, the possibility of shocks will cause an overestimation of the "true" SFR. The H $\alpha$ flux in Regions 1 and 2 is $~ 20 \%$ of the total $\mathrm{H} \alpha$ flux. Rich et al. (2010) showed that for a slow shock of $\sim 100 \mathrm{~km} \mathrm{~s}^{-1}, 50 \%-70 \%$ of the $\mathrm{H} \alpha$ luminosity can be ascribed to shocks. Hence, $\sim 10 \%$ of the total $\mathrm{H} \alpha$ flux of A2667arc may result from shocks.

\section{SUMMARY}

We present the OSIRIS IFU data analysis of the spatially resolved giant arc at $z=1.03$ in A2667. With a flux magnification of $14.0 \pm 2$ in flux and $\sim 2 \times 7$ in area, we achieved a spatial resolution of $115-405 \mathrm{pc}$ on the source plane. We find the A2667arc is clumpy in rest-frame UV and optical morphology, and has a high velocity dispersion $V_{\text {rot }} \sin i / \sigma \sim 0.73$, similar to the turbulent SF disks at $z \sim 2$. The current morphology and velocity map is not sufficient to distinguish whether the A2667 arc is a face-on clumpy disk or a merger. We find that [N $\mathrm{NI}]$ and $\mathrm{H} \alpha$ ratios first rise and then fall when moving out from the galactic center defined as the peak of $\mathrm{H} \alpha$ flux. The central $350 \mathrm{pc}$ of the $\mathrm{H} \alpha$ nucleus region is dominated by star formation with 

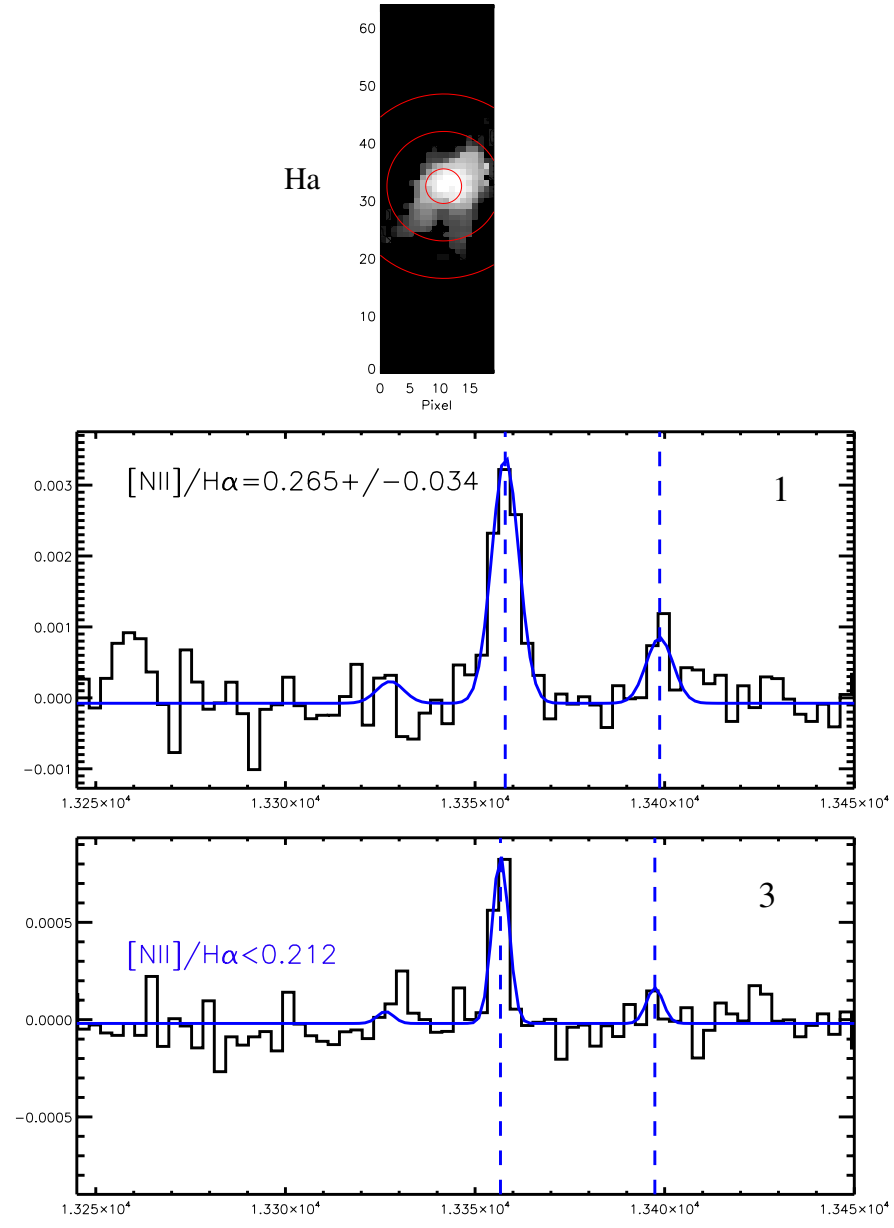
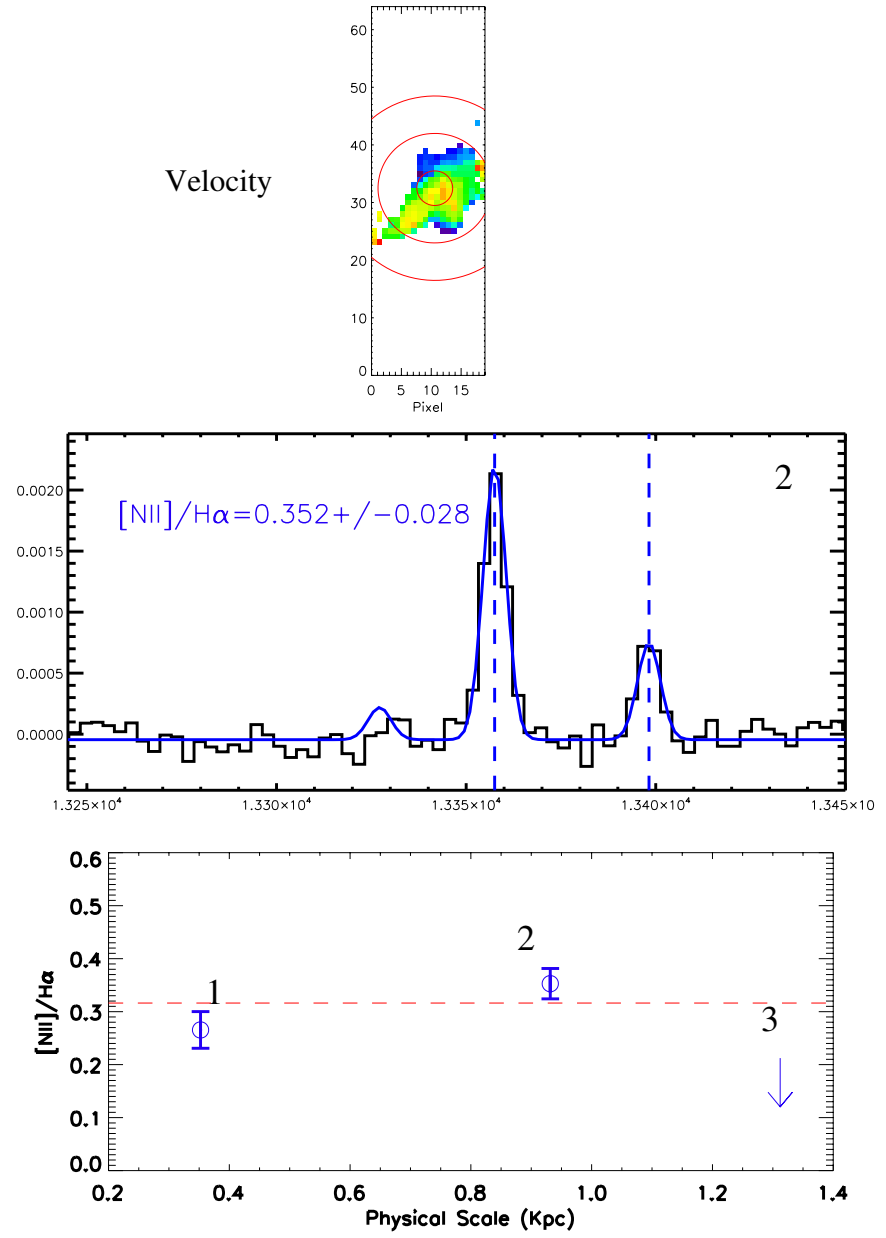

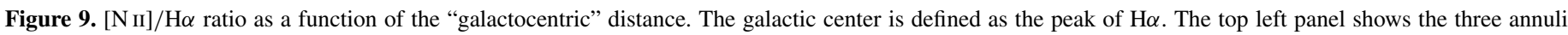

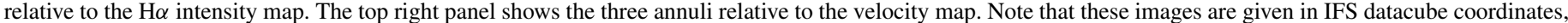

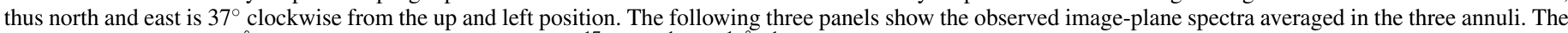

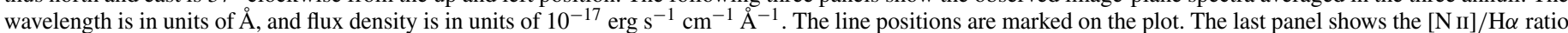

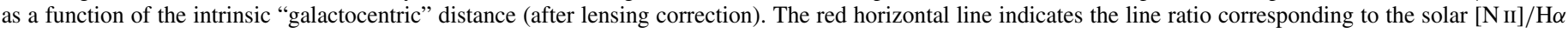
ratio. Ratios above solar may be contaminated by non-photoionized emission and cannot be directly related to metallicity.

(A color version of this figure is available in the online journal.)

metallicity $12+\log (\mathrm{O} / \mathrm{H})=8.57 \pm 0.03\left(\sim 0.76 Z_{\odot}\right)$. The $[\mathrm{N} \mathrm{II}] / \mathrm{H} \alpha$ ratios in the $350-900 \mathrm{pc}$ annulus show a significantly enhanced value ( $\sim 0.1$ dex higher than the nucleus). The highest $[\mathrm{N}$ II $] / \mathrm{H} \alpha$ ratio region $(\sim 0.2$ dex higher $)$ corresponds to a $\sim 100 \mathrm{~km} \mathrm{~s}^{-1}$ blueshifted velocity clump with respect to the nucleus. Applying the most recent slow-shock models, we propose that this elevated $[\mathrm{N} \mathrm{II}] / \mathrm{H} \alpha$ ratio is caused by shock excitation from a galactic wind. Our results indicate that extreme caution must be used when interpreting line ratios as metallicity gradients at high redshift. Shocked extranuclear regions can mimic flat or inverted metallicity gradients.

We would like to thank the referee for his/her helpful comments which have significantly improved this paper. This work is based on data obtained at the W. M. Keck Observatory. We are grateful to the Keck Observatory staff for assistance with our observations, especially Jim Lyke, Randy Campbell, and Scott Dahm. T.Y. thanks the hospitality of the Australian National University and a Founder Region Fellowship for Women. L.K. acknowledges NSF Early CAREER Award. A.M.S. acknowledges an STFC Advanced Fellowship. J.R. is supported by the Marie Curie Career Integration Grant 294074. The au- thors recognize the very significant cultural role that the summit of Mauna Kea has within the indigenous Hawaiian community.

\section{APPENDIX}

\section{[N II]/H $\alpha$ AS A FUNCTION OF RADIUS}

In metallicity gradient studies, averaging spectra in annular regions is a common exercise (Moustakas et al. 2010; Queyrel et al. 2012; Yuan et al. 2011). To relate to these studies, we extract spectra in three annuli centered on the $\mathrm{H} \alpha$ peak (Figure 9). Each spectrum is calculated as the $\mathrm{H} \alpha$ flux weighted median of all pixels within the annulus. From Section 5.2, we see that the line ratio map is not symmetric with respect to the center. Averaging spectra in annuli smooths out the lateral spatial variance, resulting in noisier [N II] lines. The choice of the annuli is such that the integrated spectra give $\mathrm{S} / \mathrm{N}>5$ for the $[\mathrm{N}$ II] lines. The physical lengths of the three outer radii of the annuli in the source plane are $0.35,0.93$, and $1.31 \mathrm{kpc}$, respectively. The $[\mathrm{N}$ II] line is robustly detected at $\mathrm{S} / \mathrm{N}>5$ for the inner two annuli and is a $3 \sigma$ detection for the outer annulus.

The $[\mathrm{N}$ II $] / \mathrm{H} \alpha$ ratios are larger in the circumnuclear region $(350-930 \mathrm{pc})$ than in the nucleus $(<350 \mathrm{pc})$. Small $[\mathrm{N} \mathrm{II}] / \mathrm{H} \alpha$ 
ratios are seen in the outer annulus $(930-1.3 \mathrm{kpc})$. If the $[\mathrm{N}$ II] $/ \mathrm{H} \alpha$ ratios arise purely from $\mathrm{H}$ II regions, these $[\mathrm{N} \mathrm{II}] / \mathrm{H} \alpha$ ratios could translate into a flat or inverted metallicity gradient similar to those in several high-redshift studies such as Cresci et al. (2010) and Queyrel et al. (2012).

However, in A2667arc, these enhanced circumnuclear $[\mathrm{N}$ II] $/ \mathrm{H} \alpha$ ratios are remarkably similar to the LINER-like luminous infrared galaxies reported in Rich et al. (2010, 2011). Rich et al. observed that enhanced extranuclear $[\mathrm{N}$ II] $/ \mathrm{H} \alpha$ ratios in late-stage galaxy mergers are driven by slow-shock emission (100-200 $\left.\mathrm{km} \mathrm{s}^{-1}\right)$ associated with galactic-wide winds or gas collisions due to mergers. Rich et al. $(2010,2011)$ showed that both velocity dispersions and the strong line ratios independently indicate shock excitation in the extranuclear regions. Slow-shock models confirm this conclusion, yielding remarkable agreement with the enhanced emission-like ratios.

\section{REFERENCES}

Alexander, D. M., Swinbank, A. M., Smail, I., McDermid, R., \& Nesvadba, N. P. H. 2010, MNRAS, 402, 2211

Allen, M. G., Dopita, M. A., \& Tsvetanov, Z. I. 1998, ApJ, 493, 571

Arribas, S., Colina, L., \& Clements, D. 2001, ApJ, 560, 160

Arribas, S., Colina, L., Monreal-Ibero, A., et al. 2008, A\&A, 479, 687

Asplund, M., Grevesse, N., \& Sauval, A. J. 2005, in ASP Conf. Ser. 336, Cosmic

Abundances as Records of Stellar Evolution and Nucleosynthesis, ed. T. G.

Barnes, III \& F. N. Bash (San Francisco, CA: ASP), 25

Baldwin, J. A., Phillips, M. M., \& Terlevich, R. 1981, PASP, 93, 5

Bower, R. G., Benson, A. J., Malbon, R., et al. 2006, MNRAS, 370, 645

Bruzual, G., \& Charlot, S. 2003, MNRAS, 344, 1000

Calzetti, D. 1997, in AIP Conf. Ser. 408, The Ultraviolet Universe at Low and High Redshift, ed. W. H. Waller (Melville, NY: AIP), 403

Calzetti, D., Armus, L., Bohlin, R. C., et al. 2000, ApJ, 533, 682

Cardelli, J. A., Clayton, G. C., \& Mathis, J. S. 1989, ApJ, 345, 245

Chabrier, G. 2003, PASP, 115, 763

Covone, G., Kneib, J.-P., Soucail, G., et al. 2006, A\&A, 456, 409

Crain, R. A., Theuns, T., Dalla Vecchia, C., et al. 2009, MNRAS, 399, 1773

Cresci, G., Mannucci, F., Maiolino, R., et al. 2010, Nature, 467, 811

Davé, R., Finlator, K., \& Oppenheimer, B. D. 2011, MNRAS, 416, 1354

Davies, R. I. 2007, MNRAS, 375, 1099

Dekel, A., Sari, R., \& Ceverino, D. 2009, ApJ, 703, 785

Denicoló, G., Terlevich, R., \& Terlevich, E. 2002, MNRAS, 330, 69

Erb, D. K., Steidel, C. C., Shapley, A. E., et al. 2006, ApJ, 646, 107

Farage, C. L., McGregor, P. J., Dopita, M. A., \& Bicknell, G. V. 2010, ApJ, 724,267

Finlator, K., \& Davé, R. 2008, MNRAS, 385, 2181

Flores, R. A., \& Primack, J. R. 1994, ApJ, 427, L1

Förster Schreiber, N. M., Genzel, R., Bouché, N., et al. 2009, ApJ, 706, 1364

Förster Schreiber, N. M., Shapley, A. E., Erb, D. K., et al. 2011, ApJ, 731, 65

Garn, T., \& Best, P. N. 2010, MNRAS, 409, 421

Genzel, R., Newman, S., Jones, T., et al. 2011, ApJ, 733, 101

Genzel, R., Tacconi, L. J., Eisenhauer, F., et al. 2006, Nature, 442, 786

Governato, F., Brook, C., Mayer, L., et al. 2010, Nature, 463, 203

Governato, F., Zolotov, A., Pontzen, A., et al. 2012, MNRAS, 422, 1231

Hopkins, P. F., Quataert, E., \& Murray, N. 2012, MNRAS, 421, 3522

Ilbert, O., Salvato, M., Le Floc'h, E., et al. 2010, ApJ, 709, 644

Jones, T., Ellis, R., Jullo, E., \& Richard, J. 2010a, ApJ, 725, L176

Jones, T. A., Swinbank, A. M., Ellis, R. S., Richard, J., \& Stark, D. P. 2010b, MNRAS, 404, 1247

Jullo, E., Kneib, J.-P., Limousin, M., et al. 2007, New J. Phys., 9, 447

Kauffmann, G., Heckman, T. M., Tremonti, C., et al. 2003, MNRAS, 346, 1055

Kennicutt, R. C., Jr. 1998, ApJ, 498, 541

Kewley, L. J., \& Dopita, M. A. 2002, ApJS, 142, 35

Kewley, L. J., Dopita, M. A., Sutherland, R. S., Heisler, C. A., \& Trevena, J. 2001a, ApJ, 556, 121

Kewley, L. J., Groves, B., Kauffmann, G., \& Heckman, T. 2006, MNRAS, 372, 961

Kewley, L. J., Heisler, C. A., Dopita, M. A., \& Lumsden, S. 2001b, ApJS, 132,37
Kirby, E. N., Martin, C. L., \& Finlator, K. 2011, ApJ, 742, L25

Kneib, J. P., Mellier, Y., Fort, B., \& Mathez, G. 1993, A\&A, 273, 367

Larkin, J., Barczys, M., Krabbe, A., et al. 2006, New Astron. Rev., 50, 362

Law, D. R., Steidel, C. C., Erb, D. K., et al. 2009, ApJ, 697, 2057

Lehnert, M. D., Nesvadba, N. P. H., Le Tiran, L., et al. 2009, ApJ, 699, 1660

Leitherer, C., Schaerer, D., Goldader, J. D., et al. 1999, ApJS, 123, 3

Levesque, E. M., Kewley, L. J., \& Larson, K. L. 2010, AJ, 139, 712

Martin, C. L. 1999, ApJ, 513, 156

Martin, C. L. 2005, ApJ, 621, 227

Martin, C. L., \& Bouché, N. 2009, ApJ, 703, 1394

Martin, C. L., Shapley, A. E., Coil, A. L., et al. 2012, arXiv:1206.5552

Moustakas, J., Kennicutt, R. C., Jr., Tremonti, C. A., et al. 2010, ApJS, 190, 233

Murray, N., Quataert, E., \& Thompson, T. A. 2005, ApJ, 618, 569

Nesvadba, N. P. H., Lehnert, M. D., Davies, R. I., Verma, A., \& Eisenhauer, F. 2008, A\&A, 479, 67

Nesvadba, N. P. H., Lehnert, M. D., Genzel, R., et al. 2007, ApJ, 657, 725

Noeske, K. G., Faber, S. M., Weiner, B. J., et al. 2007a, ApJ, 660, L47

Noeske, K. G., Weiner, B. J., Faber, S. M., et al. 2007b, ApJ, 660, L43

Oppenheimer, B. D., \& Davé, R. 2008, MNRAS, 387, 577

Osterbrock, D. E. 1989, Astrophysics of Gaseous Nebulae and Active Galactic Nuclei (Mill Valley, CA: University Science Books), 422

Pettini, M., \& Pagel, B. E. J. 2004, MNRAS, 348, L59

Pettini, M., Shapley, A. E., Steidel, C. C., et al. 2001, ApJ, 554, 981

Pilkington, K., Gibson, B. K., Calura, F., et al. 2011, MNRAS, 417, 2891

Queyrel, J., Contini, T., Kissler-Patig, M., et al. 2012, A\&A, 539, A93

Rich, J. A., Dopita, M. A., Kewley, L. J., \& Rupke, D. S. N. 2010, ApJ, 721,505

Rich, J. A., Kewley, L. J., \& Dopita, M. A. 2011, ApJ, 734, 87

Rich, J. A., Torrey, P., Kewley, L. J., Dopita, M. A., \& Rupke, D. S. N. 2012, ApJ, 753,5

Richard, J., Smith, G. P., Kneib, J.-P., et al. 2010, MNRAS, 404, 325

Rubin, K. H. R., Prochaska, J. X., Koo, D. C., \& Phillips, A. C. 2012, ApJ, 747, L26

Rubin, K. H. R., Prochaska, J. X., Koo, D. C., Phillips, A. C., \& Weiner, B. J. 2010a, ApJ, 712, 574

Rubin, K. H. R., Prochaska, J. X., Ménard, B., et al. 2011, ApJ, 728, 55

Rubin, K. H. R., Weiner, B. J., Koo, D. C., et al. 2010b, ApJ, 719, 1503

Rupke, D. S., Veilleux, S., \& Sanders, D. B. 2002, ApJ, 570, 588

Rupke, D. S., Veilleux, S., \& Sanders, D. B. 2005, ApJ, 632, 751

Salpeter, E. E. 1955, ApJ, 121, 161

Sato, T., Martin, C. L., Noeske, K. G., Koo, D. C., \& Lotz, J. M. 2009, ApJ, 696, 214

Shapiro, K. L., Genzel, R., Quataert, E., et al. 2009, ApJ, 701, 955

Shapley, A. E., Steidel, C. C., Pettini, M., \& Adelberger, K. L. 2003, ApJ, 588,65

Sharon, K., Gladders, M. D., Rigby, J. R., et al. 2012, ApJ, 746, 161

Sharp, R. G., \& Bland-Hawthorn, J. 2010, ApJ, 711, 818

Shopbell, P. L., \& Bland-Hawthorn, J. 1998, ApJ, 493, 129

Sobral, D., Best, P. N., Matsuda, Y., et al. 2012a, MNRAS, 420, 1926

Sobral, D., Smail, I., Best, P. N., et al. 2012b, in IAC Talks, Astronomy and Astrophysics Seminars from the Instituto de Astrofísica de Canarias, 291

Soto, K. T., \& Martin, C. L. 2012, arXiv:1205.0082

Soto, K. T., Martin, C. L., Prescott, M. K. M., \& Armus, L. 2012, arXiv: 1205.0083

Stark, D. P., Swinbank, A. M., Ellis, R. S., et al. 2008, Nature, 455, 775

Steidel, C. C., Erb, D. K., Shapley, A. E., et al. 2010, ApJ, 717, 289

Sutherland, R. S., Bicknell, G. V., \& Dopita, M. A. 1993, ApJ, 414, 510

Swinbank, A. M., Bower, R. G., Smith, G. P., et al. 2007, MNRAS, 376, 479

Swinbank, A. M., Smail, I., Bower, R. G., et al. 2005, MNRAS, 359, 401

Swinbank, M. 2007, in ASP Conf. Ser. 379, Cosmic Frontiers, ed. N. Metcalfe \& T. Shanks (San Francisco, CA: ASP), 226

Tremonti, C. A., Heckman, T. M., Kauffmann, G., et al. 2004, ApJ, 613, 898 van der Wel, A., Straughn, A. N., Rix, H.-W., et al. 2011, ApJ, 742, 111

Veilleux, S., Cecil, G., \& Bland-Hawthorn, J. 2005, ARA\&A, 43, 769

Veilleux, S., Kim, D.-C., \& Sanders, D. B. 2002, ApJS, 143, 315

Veilleux, S., \& Osterbrock, D. E. 1987, ApJS, 63, 295

Veilleux, S., \& Rupke, D. S. 2002, ApJ, 565, L63

Weiner, B. J., Coil, A. L., Prochaska, J. X., et al. 2009, ApJ, 692, 187

Westmoquette, M. S., Exter, K. M., Smith, L. J., \& Gallagher, J. S. 2007, MNRAS, 381, 894

Wuyts, E., Rigby, J. R., Gladders, M. D., et al. 2012, ApJ, 745, 86

Wuyts, S., Förster Schreiber, N. M., van der Wel, A., et al. 2011, ApJ, 742, 96

Yuan, T.-T., Kewley, L. J., Swinbank, A. M., Richard, J., \& Livermore, R. C. 2011, ApJ, 732, L14 